\title{
Disclosure of funding sources and conflicts of interest in evidence underpinning vitamin $D$ and calcium recommendations in bone health guidelines
}

\author{
Liora Baram¹, Zhaoli Dai ${ }^{1}$ (1), Sally McDonald ${ }^{1}$ and Lisa A Bero ${ }^{1,2, *}$ (1) \\ ${ }^{1}$ Charles Perkins Centre, School of Pharmacy, Faculty of Medicine and Health, The University of Sydney, D17, The Hub, \\ Camperdown, NSW 2006, Australia: ${ }^{2}$ School of Medicine and Colorado School of Public Health, Center for Bioethics \\ and Humanities, University of Colorado Anschutz Medical Campus, Mail Stop B137, 13080 E. 19th Ave, Aurora, \\ CO 80045, USA
}

Submitted 11 August 2021: Final revision received 16 November 2021: Accepted 13 January 2022: First published online 24 January 2022

\begin{abstract}
Objective: The present study aims to examine the relationship between study funding sources, author conflicts of interest (COI) and conclusions in studies supporting vitamin $\mathrm{D}$ and $\mathrm{Ca}$ intake cited in bone health guideline recommendations.

Design: Cross-sectional

Setting: Forty-seven global bone health guidelines with vitamin D and/or Ca recommendations for adults aged 40 years and above.

Participants: The evidence cited to support the recommendations was extracted by two independent reviewers and classified by type of recommendation, article characteristics, study design, types of funding sources and conflict of interest (COI) disclosure and direction of study conclusions.

Results: Of 156 articles cited to support the bone health recommendations, $120(77 \%)$ disclosed a funding source, and 43 (28\%) declared that at least one author had a COI. Compared with articles with non-commercial or no funding source, those funded by commercial sponsors tended to have a study conclusion favourable towards vitamin $\mathrm{D} / \mathrm{Ca}$ (relative risk (95\% CI): 1.32 (0.94, 1.87), $P=0 \cdot 16$ ), but the association was not statistically significant (Fisher's exact test). Compared to those with a COI disclosure statement, articles with missing or unclear COI disclosure were more likely to have favourable conclusions (1.56 (1.05, 2.31), $P=0.017$ ) (Fisher's exact test).

Conclusion: In the evidence underpinning a sample of global bone health guidelines, COI disclosure was low and studies with missing or unclear COI disclosures were more likely to have favourable study conclusions than those with disclosures, suggesting a need for greater transparency of COI in bone health guidelines.
\end{abstract}

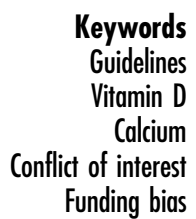

Keywords

Guidelines

Calcium

Funding bias
Studies in biomedical research, including those on tobacco $^{(1)}$, drugs or medical devices ${ }^{(2,3)}$ have found that industry funding sources and/or author financial conflicts of interest (COI) are positively associated with research outcomes that favour the products of the sponsoring industry $^{(2-6)}$. Additionally, recent studies related to foods or nutrients also suggest that authors with industry ties are more likely to find favourable health outcomes associated with the food or nutritional products investigated than those without industry ties ${ }^{(7,8)}$. Supplementation of vitamin $\mathrm{D}$ and $\mathrm{Ca}$ is common in the general population and $\mathrm{a}$ frequent topic in scientific literature. Previous analysis has found strong ties between industry and academia in Vitamin D and Ca research, suggesting a COI due to the high profitability of $\mathrm{Ca}$ and vitamin $\mathrm{D}$ supplements ${ }^{(9)}$.

Vitamin D and Ca are essential nutrients for bone health and maintenance ${ }^{(10)}$. Our findings from a systematic review of 47 global bone health guidelines suggested that recommendations for vitamin D and Ca vary substantially across the guidelines ${ }^{(11)}$. Furthermore, there has been increasing use of vitamin $\mathrm{D}^{(12)}$ and Ca supplements ${ }^{(13)}$, as well as increased testing of serum 25 hydroxyvitamin D $(25(\mathrm{OH})$ D) in the past 20 years, even among populations where sufficient vitamin D levels were found ${ }^{(14)}$, and government 
policies restricted vitamin D testing to high-risk groups ${ }^{(15)}$ and concerns about toxicity ${ }^{(16)}$. Using serum 25 hydroxyvitamin D (25(OH)D) cut-offs as a basis for recommending supplementation is further compromised by the lack of standardised vitamin D measures ${ }^{(17)}$. Increased nonstandardised testing and unnecessary recommendations for supplement intake may contribute to the overdiagnosis and overtreatment of vitamin D deficiency, which arguably is driven by commercial players.

Few studies have quantified the relationship between funding sources and/or authors' COI and study conclusions among studies focused on the bone health benefits of vitamin D or Ca. For example, one study focusing on nineteen randomised controlled trials of Ca supplementation in children found that most of the studies ( $n 16$ ) were industry funded and most had findings in favour of supplementation ( $n$ 16), and was thus not able to detect a statistical association between funding source and study outcome ${ }^{(18)}$. We hypothesised that studies with commercial funding sources and/or COI related to commercial industries would be more likely to have conclusions supporting the intake of vitamin D and/or Ca supplements and fortified foods, as compared with studies with non-commercial funding. These relationships would provide insights into the potential influence of these financial ties on vitamin $\mathrm{D}$ and $\mathrm{Ca}$ research, and further support the importance of transparency in funding and COI disclosure in evidencebased guidelines.

\section{Methods}

We conducted a cross-sectional study of articles cited in bone health guideline recommendations.

\section{Protocol and registration}

This study examines articles that were cited in bone health guidelines identified in published systematic review ${ }^{(11)}$. The peer-reviewed protocol for this systematic review was published ${ }^{(19)}$ and registered in PROSPERO (registration number: CRD42019126452) in March 2019.

\section{Data sources}

As described in our protocol, bone health guidelines were identified by searching MEDLINE (via OVID), EMBASE (via OVID), CINAHL (via EBSCO), Practice-Based Evidence in Nutrition, National Guideline Clearinghouse (by Agency for Healthcare Research and Quality, AHRQ), NICE, Guidelines International Network (GIN) and the website of the International Osteoporosis Foundation in March 2019 for guidelines published from 1 January 2009 to 28 February 2019. We identified fortyseven guidelines for inclusion in the systematic review of guidelines $^{(11)}$.
For this study, two authors independently extracted citations of articles referenced as evidence underpinning vitamin D and Ca recommendations from the forty-seven global bone health guidelines published between 2009 and February 2019. Articles referenced to support recommendations in the guidelines were screened; duplicates, non-English language papers and studies that did not directly assess vitamin D or Ca were excluded (see Fig. 1).

\section{Data extraction}

Full texts were retrieved for data extraction. Any discrepancies in the data extracted from the studies were resolved by discussion or through consultation with the senior author.

Using a pilot-tested data extraction form on the Research Electronic Data Capture (REDCap) ${ }^{(20)}$ hosted at The University of Sydney, two reviewers independently extracted data from the full text of each piece of evidence cited in the guidelines. Table 1 shows the type of data collected in each of the following categories: article characteristics, type of guideline recommendation, study design, funding source disclosure, author COI disclosure, types of funding source and author COI and study conclusions.

\section{Coding of funder and COI information}

For funding sources, we first identified whether a funding disclosure was present or absent; if present, verbatim text and information about the funders were extracted and categorised as commercial, non-commercial or unclear (Table 1). In cases where the financing of the organisation was difficult to determine, we searched the internet to gather information on the organisation.

The entire COI declaration, if present, was extracted verbatim. Where COI statements were not found in a designated section of the article, a search of key terms such as 'conflict', 'interest', 'declare', 'disclose', was used to locate COI declarations in the main text. Similar to the categorisation of funders, we categorised the COI statements as: No (explicit statement that the authors had no COI); Missing COI declaration; Uncertain (it was not clear whether a COI was being declared) and Declared COI (at least one author declared a COI).

\section{Coding of autbors' COI}

Where a COI declaration was found, we extracted information about each of the authors for whom a COI was declared. Author information extracted included Name and Author Order (for example, 1st, 2nd, 3rd ... Last). We counted the number of authors that declared commercial COI, no COI or did not have a disclosure, using the approach mentioned above.

\section{Classification of study conclusions}

Study conclusions were extracted from the full text of the article, for example, in the discussion or conclusion section (as opposed to abstracts). Where one or more conclusions 


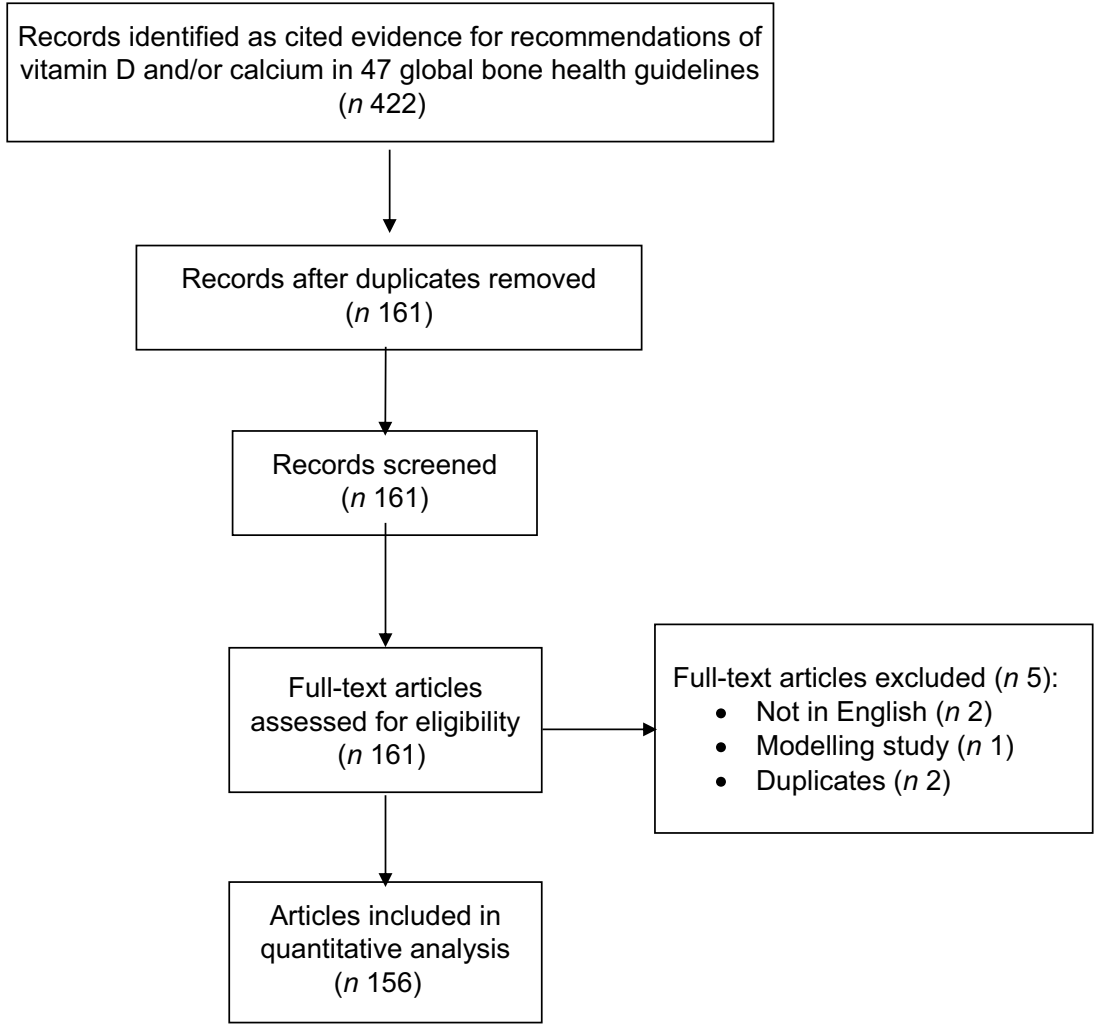

Fig. 1 Flow chart of study selection

favoured the intervention or exposure investigated in the study, we classified the article as having a favourable study conclusion. Where the conclusion recommended against an intervention or exposure, we classified this as unfavourable. Neutral conclusions were classified as those which did not clearly conclude in either a favourable or unfavourable direction. We combined unfavourable and neutral conclusions as one category for analysis.

\section{Data synthesis and analysis}

Descriptive statistics were used to summarise article characteristics by funding source and author COI. We assessed the relationship of presence of funding and COI disclosures, and types of funding or COI with the direction of a study conclusion (i.e. favourable towards the intervention being tested or not), using the Fisher's exact test to estimate relative risk (RR) (95\% CI) and test of significance. We used Fisher's exact test because of the small sample size. Specifically, we analysed the following relationships: (1) funding disclosure, (2) type of funding category, (3) COI disclosure and (4) Missing or unclear COI statement $v$. presence of COI. Our primary outcome was defined as a study conclusion in favour of vitamin D and/or Ca.

Our primary analyses examining the association of funding source and COI with article conclusions was the entire sample of articles cited to support the recommendations ( $n$ 156). We performed a sensitivity analysis limited to the sample of studies focusing on vitamin $\mathrm{D}$ and/or Ca supplements ( $n$ 103). As we observed variability in the journal impact factors and citation rates for the articles in our sample, we also conducted a post hoc analysis of the association of journal impact factor and study citation with study conclusion, disclosure of funding source and disclosure of COI for the full sample ( $n$ 156). A two-sided $P$-value less than 0.05 was considered statistically significant. All data analyses were conducted in SAS 9.4.

\section{Results}

Among the 422 articles cited as evidence for recommendations of vitamin $\mathrm{D}$ and/or Ca in the 47 bone health guidelines, we included a final sample of 156 articles (Fig. 1).

\section{Article characteristics}

Characteristics of all included articles ( $n$ 156) are described in Table 2. The publication period spans from 1985 to 2018. The mean 5-year impact factor is $15 \cdot 7$, with $53 / 156$ articles (34\%) coming from journals with an impact factor between 19.658 and 59.245. Two-thirds of the articles (104/156) have over 100 citations, and one-third (52/156) have been cited between 338 and 851 times. Thirty-nine (39/156, $27 \%)$ articles are systematic reviews; 49 (49/156, $31 \%)$ are randomised control trials (RCT). Over $75 \%(120 / 156)$ of the articles disclose funding sources, while $19 \%$ (29/156) do not have a statement disclosing funding 
Table 1 Data extraction elements

\begin{tabular}{|c|c|}
\hline Category & Items \\
\hline Article characteristics & $\begin{array}{l}\text { Names and numbers of } \\
\text { authors } \\
\text { Title of study } \\
\text { Year of publication } \\
\text { Published journal } \\
5 \text {-year journal impact factor } \\
\text { Number of times article is } \\
\text { cited }\end{array}$ \\
\hline $\begin{array}{l}\text { Types of guideline } \\
\text { recommendation }\end{array}$ & $\begin{array}{l}\text { Dietary vitamin } D \\
\text { Vitamin } D \text { supplements } \\
\text { Recommended serum } \\
25(\mathrm{OH}) \mathrm{D} \text { level } \\
\text { Vitamin D from sun exposure } \\
\text { Dietary Ca } \\
\text { Ca supplements }\end{array}$ \\
\hline Study design & $\begin{array}{l}\text { Randomised control trial } \\
\text { (RCT) Non-randomised trial } \\
\text { Cohort study } \\
\text { Cross-sectional study } \\
\text { Case control study } \\
\text { Systematic review of RCT } \\
\text { Systematic review of } \\
\text { non-RCT } \\
\text { Others (e.g. opinion, } \\
\text { commentary, editorial, } \\
\text { narrative review) }\end{array}$ \\
\hline Funding source disclosure & $\begin{array}{l}\text { Disclosed funding source } \\
\text { Disclosed no funding source } \\
\text { Disclosure missing } \\
\text { Unclear }\end{array}$ \\
\hline $\begin{array}{l}\text { Author conflict of interest (COI) } \\
\text { disclosure }\end{array}$ & $\begin{array}{l}\text { No COI (explicitly stating } \\
\text { none) } \\
\text { Missing COI declaration } \\
\text { Uncertain } \\
\text { Declared COI in at least one } \\
\text { author }\end{array}$ \\
\hline $\begin{array}{l}\text { Funding source/COI by } \\
\text { commercial funding category }\end{array}$ & $\begin{array}{l}\text { Supplement } \\
\text { Pharmaceutical } \\
\text { Dairy } \\
\text { Food } \\
\text { Non-dairy } \\
\text { Tanning industry } \\
\text { Other }\end{array}$ \\
\hline $\begin{array}{l}\text { Funding source/COI by non- } \\
\text { commercial funding category }\end{array}$ & $\begin{array}{l}\text { University } \\
\text { Government } \\
\text { Other non-profit } \\
\text { Charity/philanthropic } \\
\quad \text { organisation }\end{array}$ \\
\hline Direction of study conclusion & $\begin{array}{l}\text { Favourable to vitamin } \mathrm{D} / \mathrm{Ca} \\
\text { Unfavourable to vitamin } \mathrm{D} / \mathrm{Ca} \\
\text { Neutral about vitamin } \mathrm{D} / \mathrm{Ca}\end{array}$ \\
\hline
\end{tabular}

sources. Around $40 \%$ of the articles (62/156) explicitly state that the authors have no COI, while $31 \%$ (49/156) do not have a COI declaration of any sort. The majority of articles (66\%, 103/156) were cited to support recommendations for use of $\mathrm{Ca}$ or vitamin $\mathrm{D}$ supplements. As shown in Table 2, the characteristics of the subset of articles on supplements ( $n$ 103) were similar to the full sample.

Sources of funding and COI are described in Table 3. Among the articles that declared any kind of funding source ( $n$ 120), 34 (28.3\%) declared receiving partial or full funding from commercial sponsors, and 113 (94.2\%) declared non-commercial sources. Pharmaceutical companies were the major commercial sponsors ( 18 of 120 articles
(15.0\%)), and governments were the major non-commercial sponsors (79 of $120(65.8 \%)$ articles). Among the authors that declared any kind of COI ( $n$ 140), 139 (99.3\%) declared at least one commercial COI, and 44 (31.4\%) declared at least one non-commercial COI. Pharmaceutical industry sources were the most common commercial COI (97 of 139 (69.8\%) authors), and university sources were the most common non-commercial COI (29 of $44(65.9 \%)$ authors).

There were a number of funding sources and COI that did not fit into our established categories or were ambiguous. These included funding entities such as boards, professional societies, councils and 'alliances' that appeared to be affiliated with industry but claimed to be non-profit. Examples for 'unclear' or 'other commercial' funders are shown in Appendix 1.

\section{Association between funding source/autbor COI and study conclusion}

Of 156 included articles, 76 (48.7\%) had conclusions that we classified as favourable towards the use of vitamin D or Ca (Table 4). Articles that disclosed any funding source ( $n$ 120), compared with articles that disclosed they had no funding, or had a missing or unclear funding source ( $n$ 36), were more likely to have conclusions in favour of vitamin $\mathrm{D}$ or Ca (RR: $1.45(0.91,2.32), P=0.09)$, although the difference was not statistically significant. Articles that were sponsored by a commercial organisation ( $n$ 34), compared with articles that were sponsored by non-commercial funders or those without funding source ( $n 90)$, tended to have conclusions in favour of vitamin D/Ca (RR: 1.32 (0.94, 1.87), $P=0 \cdot 16$ ), but these results are not statistically significant.

As shown in Table 4 , articles with a missing or unclear COI disclosure had a 0 -4-fold higher risk of having a favourable conclusion compared with those that declared no COI (RR: $1.39(0.98,1.96), P=0.09)$. The likelihood of having a favourable conclusion was even higher when we compared studies with missing or unclear COI disclosures with those with presence of a COI disclosure statement (RR: $1.56(1.05,2 \cdot 31), P=0.017)$.

Sensitivity analyses were conducted by limiting the analyses to the studies focusing on vitamin $\mathrm{D} / \mathrm{Ca}$ supplements ( $n$ 103). We found results similar to those of the whole sample (online supplementary material, Supplemental Table 1).

The analysis of the association of journal impact factors and studies' citations with (1) study conclusion, (2) disclosure of funding source and (3) disclosure of COI (online supplementary material, Supplemental Table 2) shows that a higher impact factor or higher citations was associated with a higher likelihood of disclosed funding source $(P<0.05)$. However, study citation was inversely associated with disclosure of COI $(P<0.05)$. There was no significant association of journal impact factor or article citations with conclusions favouring the intervention. 
Table 2 Characteristics of articles cited as evidence to support vitamin D and/or Ca recommendations in forty-seven bone health guidelines

\begin{tabular}{|c|c|c|c|c|}
\hline & \multicolumn{2}{|c|}{ All $(n 156)$} & \multicolumn{2}{|c|}{$\begin{array}{l}\text { Articles on vitamin } \mathrm{D} / \mathrm{Ca} \\
\text { supplements }(n 103)\end{array}$} \\
\hline & $n$ & $\%$ & $n$ & $\%$ \\
\hline \multicolumn{5}{|l|}{ Publication year } \\
\hline $1985-2009$ & 85 & $54 \cdot 5$ & 58 & $56 \cdot 3$ \\
\hline 2010-2018 & 71 & 45.5 & 45 & 43.7 \\
\hline \multicolumn{5}{|l|}{ Journal 5-year impact factor* } \\
\hline Low: $3.485(3.285-3.917)$ & 51 & $32 \cdot 7$ & 27 & $26 \cdot 2$ \\
\hline Medium: 5.985 (5.879-7.831) & 52 & 33.3 & 30 & $29 \cdot 1$ \\
\hline High: 27.997 (19.658-59.245) & 53 & $34 \cdot 0$ & 46 & 44.7 \\
\hline \multicolumn{5}{|l|}{ Frequency of citations* ${ }^{*}$} \\
\hline Low: Tertile 1: 34.5 (19-53) & 52 & 33.3 & 34 & $33 \cdot 0$ \\
\hline Medium: Tertile 2: 138 (105-192) & 52 & 33.3 & 35 & $34 \cdot 0$ \\
\hline High: Tertile 3: 523.5 (338.5-851.2) & 52 & 33.3 & 34 & 33.0 \\
\hline \multicolumn{5}{|l|}{ Study design } \\
\hline RCT & 49 & 31.4 & 44 & 43.6 \\
\hline Non-randomised trial & 2 & 1.3 & 1 & 0.99 \\
\hline Cohort study & 19 & $12 \cdot 2$ & 7 & $6 \cdot 9$ \\
\hline Cross-sectional study & 18 & 11.5 & 6 & $5 \cdot 9$ \\
\hline Case control study & 1 & 0.6 & 1 & 0.99 \\
\hline Systematic review of RCT & 36 & 25 & 32 & $31 \cdot 1$ \\
\hline Systematic review of non-RCT & 3 & 1.9 & 1 & 0.99 \\
\hline Commentary/editorial/narrative review & 28 & $17 \cdot 9$ & 11 & $10 \cdot 7$ \\
\hline \multicolumn{5}{|l|}{ Funding disclosure } \\
\hline Missing & 29 & $18 \cdot 6$ & 15 & $14 \cdot 6$ \\
\hline Yes, disclosed funding sources & 120 & $76 \cdot 9$ & 85 & 82.5 \\
\hline Yes, disclosed no funding & 4 & $2 \cdot 6$ & 3 & 2.9 \\
\hline Unclear & 3 & 1.9 & 0 & \\
\hline \multicolumn{5}{|l|}{ COI disclosure } \\
\hline No (explicitly stating no COI) & 62 & 39.7 & 37 & 35.9 \\
\hline Missing COI declaration & 49 & 31.4 & 35 & $34 \cdot 0$ \\
\hline Unclear & 2 & 1.3 & 2 & 1.9 \\
\hline Declared COI in at least one author & 43 & $27 \cdot 6$ & 29 & $28 \cdot 2$ \\
\hline
\end{tabular}

$\mathrm{RCT}$, randomised control trial; COI, conflicts of interest.

*Tertiles are expressed as median (interquartile range).

\section{Discussion}

\section{Main findings}

In a set of 156 articles underpinning vitamin $\mathrm{D}$ and Ca recommendations in 47 bone health guidelines, more than $31 \%$ (49/156) of articles were missing an author COI declaration and 19\% (29/156) were missing funding disclosures. The majority of disclosed commercial funding sources (18/34) and COI (97/139) were with the pharmaceutical industry. Although we did not find a significant association between the specific commercial sponsors and article conclusions that favoured the sponsor's product, we found that articles with missing or unclear author COI disclosures were more likely to have study conclusions in favour of vitamin D or Ca compared with studies with a COI disclosure statement.

The majority of the recommendations in the bone guidelines promoted the use of vitamin $\mathrm{D}$ and $\mathrm{Ca}$ supplements rather than sunlight or food sources of vitamin $\mathrm{D}$ and $\mathrm{Ca}^{(11)}$. Thus, it is not surprising that the pharmaceutical and supplement industries were the major commercial sponsors or COI among studies that had disclosures.

\section{Implications/context of other research}

Lack of transparency

Our findings suggest that a lack of transparency about funding sources and COI in research on vitamin D and Ca characterises the evidence underlying recommendations promoting vitamin D or Ca for bone health. The lack of disclosure is particularly problematic given that most of the articles are systematic reviews or randomised controlled trials. Articles from journals with higher impact factors were more likely to have funding disclosures, suggesting that the higher impact journals may have more stringent policies or enforcement regarding disclosure of funding sources. However, all journals in the sample needed improvement in COI disclosure.

The finding that a lack of disclosure is associated with study conclusions that favour vitamin D and Ca suggests that there may be undisclosed contributors to bias in these studies. Consistent with our findings, Cherla and colleagues described a similar relationship between medical research articles with partially or undisclosed COI and favourable study conclusions, compared with those with an explicit statement of no $\mathrm{COI}^{(21)}$. In a study examining the 
Table 3 Types of funding source(s) or conflicts of interest (COI) declared

\begin{tabular}{|c|c|c|}
\hline \multirow[b]{2}{*}{ Funding category } & \multicolumn{2}{|c|}{$\begin{array}{l}\text { Study funding } \\
\text { source }(s)^{\star} n 120\end{array}$} \\
\hline & $n$ & $\%$ \\
\hline \multicolumn{3}{|l|}{ Commercial (n 34) } \\
\hline Supplement & 4 & 3 \\
\hline Pharmaceutical & 18 & 15 \\
\hline Dairy & 5 & 4 \\
\hline Food (non-dairy) & 3 & 2 \\
\hline Tanning Industry & 0 & 0 \\
\hline Others & 4 & 3 \\
\hline \multicolumn{3}{|l|}{ Non-commercial (n 113) } \\
\hline University & 20 & 17 \\
\hline Government & 79 & 66 \\
\hline Other non-commercial & 10 & 8 \\
\hline Charity & 4 & 3 \\
\hline \multirow[t]{2}{*}{ Unclear source $(n 3)$} & 3 & 2 \\
\hline & \multicolumn{2}{|c|}{$\begin{array}{l}\text { Authors declaring } \\
\text { COI† } n 140\end{array}$} \\
\hline COI category & $n$ & $\%$ \\
\hline \multicolumn{3}{|l|}{ Commercial (n 139) } \\
\hline Supplement & 4 & 3 \\
\hline Pharmaceutical & 97 & 69 \\
\hline Dairy & 9 & 6 \\
\hline Food (non-dairy) & 3 & 2 \\
\hline Tanning Industry & 3 & 2 \\
\hline Others & 23 & 16 \\
\hline Non-commercial ( $n$ 44) & 4 & 3 \\
\hline University & 29 & 21 \\
\hline Government & 8 & 6 \\
\hline Other non-commercial & 0 & 0 \\
\hline Charity & 3 & 2 \\
\hline Unclear source $(n 3)$ & 3 & 2 \\
\hline
\end{tabular}

${ }^{*}$ Denominator is articles $(n 120)$ that declared funding source; articles could have multiple declared funding sources.

†Denominator is authors $(n 140)$ that declared a COI.

association of financial links to the indoor tanning industry and conclusions of published studies on indoor tanning, an absence of a COI declaration or funding statement was considered the same as an active declaration of no conflicts or funding ${ }^{(4)}$. We argue that distinguishing between explicit declarations of no COI and missing COI declarations is important in order to transparently evaluate the potential impact of COI on study conclusions.
Transparency of funding and COI in bone health research is further obfuscated by industry support for not-for-profit organisations ${ }^{(9)}$. For example, we identified certain funding entities such as boards, professional societies, councils and 'alliances' that appear to be affiliated with industry but claimed to be non-profit. These examples include: the 'National Dairy Promotion and Research Board' (while this body sits under the US Department of Agriculture, it is comprised of industry members2020) ${ }^{(22)}$, 'The Egg Nutrition Council' (a body of experts that provide nutrition and health information to the Australian Egg Corporation Limited and have previously sponsored the Dietitians Association of Australia (now Dietitians Australia) 2121) (23), 'The Vitamin D Society' (a non-profit vitamin D advocacy organisation) ${ }^{(4)}$ and the 'UV Foundation' (non-profit arm of the Indoor Tanning Association ${ }^{(24)}$. Previous evidence has suggested a risk of bias posed by trade associations which influenced research agendas that favour industry interests ${ }^{(25)}$. Grey and Bolland have described the influence of non-profit advocacy organisations such as the International Osteoporosis Foundation, which received more than half of its sponsorship from corporate bodies with financial interests in bone research ${ }^{(9)}$.

In our study, $21 \%$ of the author COI statements disclosed government-related service, such as committee service. It has been argued that such government service does not represent a COI relevant to making recommendations to use commercial products. Rather, government service can be considered a demonstration of expertise. The importance of reflexivity and relevant reporting of conflicts have been suggested as ways to manage commercial COI and non-commercial interests ${ }^{(26)}$. Grundy and others have proposed strategies to improve accessibility, completeness, clarity and accuracy regarding COI declaration to address the importance of standardised COI reporting ${ }^{(27)}$. Future research should investigate whether disclosure of government ties is indicative of efforts to obfuscate genuine COI. Such results could provide insight into the extent and implications of this practice.

Table 4 Relative risk $(95 \% \mathrm{Cl}$ ) for study conclusion in favour of vitamin $\mathrm{D}$ and/or $\mathrm{Ca}$ associated with funding source and conflicts of interest (COI) disclosure

\begin{tabular}{|c|c|c|c|c|}
\hline All studies ( $n$ 156) & Conclusion in favour of intervention ( $n$ 76) & Relative risk & $95 \% \mathrm{Cl}$ & $P$-value \\
\hline \multicolumn{5}{|l|}{ Funding disclosure } \\
\hline Missing, unclear or no disclosure $(n 36)$ & 13 & & Ref & \\
\hline Disclosed funding sources ( $n 120)$ & 63 & 1.45 & $0.91,2.32$ & 0.09 \\
\hline \multicolumn{5}{|l|}{ Type of funding category } \\
\hline Non-commercial ( $n$ 86) and no funding $(n 4)$ & 42 & & Ref & \\
\hline Commercial funding $(n 34)$ & 21 & 1.32 & $0.94,1.87$ & $0 \cdot 16$ \\
\hline Missing, or unclear funding source ( $n$ 32) & 13 & 0.87 & 0.54 .1 .40 & 0.68 \\
\hline \multicolumn{5}{|l|}{ COI disclosure } \\
\hline No COI (explicit statement) ( $n$ 62) & 28 & & Ref & \\
\hline Missing or unclear $\mathrm{COI}$ ( $n$ 51) & 32 & $1 \cdot 39$ & $0.98,1.96$ & 0.09 \\
\hline At least one author declared $\mathrm{COI}(n 43)$ & 16 & 0.83 & $0.51,1.32$ & 0.43 \\
\hline Presence of COI disclosure statement ( $n$ 105) & 44 & & Ref & \\
\hline Missing or unclear COI disclosure statement $(n 51)$ & 32 & 1.56 & $1 \cdot 05,2 \cdot 31$ & 0.017 \\
\hline
\end{tabular}




\section{Funding of Vitamin $D$ and Ca research}

Our findings show that a large proportion of studies about vitamin $\mathrm{D}$ and/or $\mathrm{Ca}$ interventions receive government funding. This is encouraging as it challenges notions that government does not adequately fund nutrition research and that nutrition research must rely on industry funding ${ }^{(28)}$. However, the pharmaceutical industry was found to be the main source of commercial funding and author COI. This is problematic for two reasons. First, pharmaceutical industry funding might stimulate researchers to study pharmaceutical interventions rather than food- or sun-based interventions. Thus, the pharmaceutical industry could be driving the bone health research agenda, and subsequent guideline recommendations, by producing an evidence base that is oriented towards supplementation $^{(25)}$. Second, there is existing concern around a focus on supplementation in relation to overprescribing and over testing $^{(9,29-32)}$. For instance, the International Osteoporosis Foundation partnered with DSM, a multi-corporation of health, nutrition and materials and identified a global need for vitamin D supplementation based on claims of widespread deficiency ${ }^{(9,33)}$.

\section{Strengths and limitations}

The sample of articles we selected is limited to articles that were cited as evidence to support bone guideline recommendations. As a result, this may not fully capture all studies on the topic of bone health benefits of vitamin D and $\mathrm{Ca}$. On the other hand, this sample of articles is currently being used to inform clinical practice and recommendations of vitamin D and/or Ca and, thus, comprise a clinically relevant and influential collection of studies related to bone health. We relied on COI disclosed in the published articles alone and did not further investigate sources to reveal undisclosed funding sources. Hence, we cannot completely rule out cases in which some authors had incomplete or inaccurate disclosures. Low levels of disclosure have been encountered ${ }^{(5,8,34,35)}$ and demonstrated $^{(21,36)}$ in other studies and point to a broader concern regarding adherence to journal COI disclosure policies. Low levels of disclosure also meant our sample size for comparing types of COI was relatively small, and as such, our analyses lacked statistical power to detect significant differences between commercial and non-commercial COI in relation to the direction of study conclusion. As an earlier study also pointed out ${ }^{(37)}$, large sample size is needed to test the certainty of these results.

\section{Conclusion}

In this cross-sectional analysis of evidence underpinning recommendations on vitamin $\mathrm{D}$ and $\mathrm{Ca}$ in global bone health guidelines, COI disclosure is generally low. Notably, lack of COI disclosure is associated with study conclusions that favour intake of vitamin D and/or Ca. These results, along with previous studies, demonstrate the importance of transparency and standardised disclosure of conflict of interest in biomedical research.

\section{Acknowledgements}

Financial support: This work was funded Australian National Health and Medical Research Council (NHMRC) project grant APP1139997 (Bero). The funding source played no role in the conceptualisation, design, analytical methods, data interpretation, reporting of the manuscript or publication decisions. SM is a PhD student funded by the Country Women's Association (NSW) and Edna Winifred Blackman Postgraduate Research Scholarship. Conflict of interest: None for L.B., Z.D., SM, LAB. Authorship: Conceptualisation of the study: Z.D., L.A.B.; study design: L.B., Z.D., L.A.B.; data collection: L.B., Z.D.; coding: L.B., Z.D., S.M.; data analysis: L.B., Z.D., L.A.B.; first drafts of the paper: L.B., Z.D., L.A.B.; all authors critically revised the paper and approved the final draft. Ethics of human subject participation: This research did not involve human subjects and is except from ethics approval according to the University of Sydney ethical guidelines.

\section{Supplementary material}

For supplementary material/s referred to in this article, please visit https://doi.org/10.1017/S1368980022000246

\section{References}

1. Barnes DE \& Bero LA (1998) Why review articles on the health effects of passive smoking reach different conclusions. JAMA 279, 1566-1570.

2. Jorgensen AW, Maric KL, Tendal B et al. (2008) Industrysupported meta-analyses compared with meta-analyses with non-profit or no support: differences in methodological quality and conclusions. BMC Med Res Methodol 8, 60.

3. Lundh A, Lexchin J, Mintzes B et al. (2017) Industry sponsorship and research outcome. Cochrane Database Syst Rev $\mathbf{2}$, MR000033.

4. Adekunle L, Chen R, Morrison L et al. (2020) Association between financial links to indoor tanning industry and conclusions of published studies on indoor tanning: systematic review. BMJ 368, m7.

5. Amiri AR, Kanesalingam K, Cro S et al. (2014) Does source of funding and conflict of interest influence the outcome and quality of spinal research? Spine J 14, 308-314.

6. Barnes DE \& Bero LA (1996) Industry-funded research and conflict of interest: an analysis of research sponsored by the tobacco industry through the Center for Indoor Air Research. J Health Polit Policy Law 21, 515-542.

7. Chartres N, Fabbri A \& Bero LA (2016) Association of industry sponsorship with outcomes of nutrition studies: a systematic review and meta-analysis. JAMA Intern Med 176, 1769-1777. 
8. Chartres N, Fabbri A, McDonald S et al. (2020) Association of food industry ties with findings of studies examining the effect of dairy food intake on cardiovascular disease and mortality: systematic review and meta-analysis. BMJ Open 10, e039036.

9. Grey A \& Bolland M (2015) Web of industry, advocacy, and academia in the management of osteoporosis. BMJ $\mathbf{3 5 1}$, h3170.

10. International Osteoporosis Foundation (2010) Facts and statistics. Epidemiol Osteoporos 20, 1-7.

11. Dai Z, McKenzie JE, McDonald S et al. (2021) Assessment of the methods used to develop vitamin D and calcium recommendations - a systematic review of bone health guidelines. Nutrients 13, 2423.

12. Rooney MR, Harnack L, Michos ED et al. (2017) Trends in use of high-dose vitamin D supplements exceeding 1000 or 4000 International Units Daily, 1999-2014. JAMA 317, $2448-2450$.

13. Rooney MR, Michos ED, Hootman KC et al. (2018) Trends in calcium supplementation, National Health and Nutrition Examination Survey (NHANES) 1999-2014. Bone 111, 23-27.

14. Galior K, Ketha H, Grebe $\mathrm{S}$ et al. (2018) 10 years of 25-hydroxyvitamin-D testing by LC-MS/MS-trends in vitamin-D deficiency and sufficiency. Bone Rep 8, 268-273.

15. Gordon L, Waterhouse M, Reid IR et al. (2020) The vitamin D testing rate is again rising, despite new MBS testing criteria. Med J Aust 213, 155-155.e151.

16. Fraser DR (2021) Vitamin D toxicity related to its physiological and unphysiological supply. Trends Endocrinol Metab 32, 929-940.

17. Sempos CT \& Binkley N (2020) 25-Hydroxyvitamin D assay standardisation and vitamin D guidelines paralysis. Public Health Nutr 23, 1153-1164.

18. Nkansah N, Nguyen T, Iraninezhad H et al. (2009) Randomized trials assessing calcium supplementation in healthy children: relationship between industry sponsorship and study outcomes. Public Health Nutr 12, 1931-1937.

19. Dai Z, Kroeger CM, McDonald S et al. (2019) Methodological quality of public health guideline recommendations on vitamin D and calcium: a systematic review protocol. BMJ Open 9, e031840.

20. Harris PA, Taylor R, Thielke R et al. (2009) Research electronic data capture (REDCap) - a metadata-driven methodology and workflow process for providing translational research informatics support. J Biomed Inform 42, 377-381.

21. Cherla DV, Viso CP, Holihan JL et al. (2019) The effect of financial conflict of interest, disclosure status, and relevance on medical research from the United States.J Gen Intern Med 34, 429-434.

22. National Dairy Promotion \& Research Board (2020) Agricultural Marketing Service. https://www.ams.usda. gov/rules-regulations/research-promotion/dairy (accessed December 2020).
23. Simon M (2015) Is the Dieticians Association of Australia in the Pocket of Big Food? Australian Edition. http://www. eatdrinkpolitics.com/2015/02/17/is-the-dietitians-associationof-australia-in-the-pocket-of-big-food/ (accessed June 2021).

24. UV Foundation (2020) Increasing Public Awareness on th eBiologic Effects of UV Light. https://www.megasun.co.nz/ Knowledge-Centre/UV-Foundation/ (accessed December 2020).

25. Fabbri A, Lai A, Grundy Q et al. (2018) The influence of industry sponsorship on the research agenda: a scoping review. Am J Public Health 108, e9-e16.

26. Bero LA \& Grundy Q (2016) Why having a (nonfinancial) interest is not a conflict of interest. PLOS Biol 14, e2001221.

27. Grundy Q, Dunn AG \& Bero L (2020) Improving researchers' conflict of interest declarations. BMJ 368, m422.

28. Nestle M (2016) Corporate funding of food and nutrition research: science or marketing? JAMA Intern Med 176, 13-14.

29. Jarvinen TL, Michaelsson K, Jokihaara J et al. (2015) Overdiagnosis of bone fragility in the quest to prevent hip fracture. BMJ 350, h2088.

30. Johansson M, Bero L, Bonfill X et al. (2019) Cochrane sustainable healthcare: evidence for action on too much medicine. Cochrane Database Syst Rev 12, ED000143.

31. Mintzes B, Swandari S, Fabbri A et al. (2018) Does industry-sponsored education foster overdiagnosis and overtreatment of depression, osteoporosis and over-active bladder syndrome? An Australian cohort study. BMJ Open 8, e019027.

32. Moynihan R, Nickel B, Hersch J et al. (2015) Public opinions about overdiagnosis: a national community survey. PLOS ONE 10, e0125165.

33. Wahl DA, Cooper C, Ebeling PR et al. (2012) A global representation of vitamin D status in healthy populations. Arch Osteoporos 7, 155-172.

34. Bero L, Oostvogel F, Bacchetti P et al. (2007) Factors associated with findings of published trials of drug-drug comparisons: why some statins appear more efficacious than others. PLoS Med 4, e184.

35. Janssen SJ, Bredenoord AL, Dhert W et al. (2015) Potential conflicts of interest of editorial board members from five leading spine journals. PLOS ONE 10, e0127362.

36. Olavarria OA, Holihan JL, Cherla D et al. (2017) Comparison of conflicts of interest among published hernia researchers self-reported with the centers for medicare and medicaid services open payments database. J Am Coll Surg 224, 800-804.

37. Chartres N, Fabbri A, McDonald S et al. (2019) Association of industry ties with outcomes of studies examining the effect of wholegrain foods on cardiovascular disease and mortality: systematic review and meta-analysis. BMJ Open 9, $\mathrm{e} 022912$. 